\title{
Distinct Roles of Kv1 and Kv3 Potassium Channels at the Calyx of Held Presynaptic Terminal
}

\author{
Taro Ishikawa, Yukihiro Nakamura, Naoto Saitoh, Wen-Bin Li, Shinichi Iwasaki, and Tomoyuki Takahashi \\ Department of Neurophysiology, University of Tokyo Graduate School of Medicine, Tokyo 113-0033, Japan
}

Despite identification of $>100$ potassium channel subunits, relatively little is known about their roles in synaptic transmission. To address this issue we recorded presynaptic potassium currents $\left(I_{\mathrm{PK}}\right)$ directly from the calyx of Held terminal in brainstem slices of rats. $I_{\mathrm{PK}}$ was composed of a 4-aminopyridine (4-AP)-sensitive component and a smaller 4-AP-insensitive component composed of an iberiotoxin-sensitive current and an unidentified slowly activating potassium current. $I_{\mathrm{PK}}$ could also be separated into a tetraethylammonium (TEA; $1 \mathrm{mM}$ )-sensitive high-voltage-activated component and a margatoxin (10 nM)-sensitive low-voltage-activated component, which was also blocked by dendrotoxin-I (200 nм) and tityustoxin-K $\alpha(100 \mathrm{nM})$. In outside-out patches excised from calyceal terminals, TEA (1 mM) consistently and to a large extent attenuated $I_{\mathrm{PK}}$, whereas margatoxin attenuated $I_{\mathrm{PK}}$ only in a subset of patches (three of seven). Immunocytochemical examination using Kv subtype-specific antibodies indicated that multiple Kv1 and Kv3 subtypes were present at the calyceal terminal. In paired presynaptic and postsynaptic whole-cell recordings, TEA (1 mM) increased both the duration and peak amplitude of presynaptic action potentials and simultaneously potentiated EPSCs. Margatoxin alone had no such effect but reduced the amount of depolarization required for action potential generation, thereby inducing a burst of spikes when the nerve terminal was depolarized for a prolonged period. Thus, at the calyx of Held terminal, Kv3 channels directly regulate evoked transmitter release, whereas $\mathrm{Kv} 1$ channels reduce nerve terminal excitability, thereby preventing aberrant transmitter release. We conclude that both $\mathrm{Kv} 3$ and Kv1 channels contribute differentially to maintaining the fidelity of synaptic transmission at the calyx of Held.

Key words: potassium channel; calyx of Held; transmitter release; Kv1; Kv3; TEA; margatoxin; HVA; LVA

\section{Introduction}

Potassium channel blockers increase transmitter release by broadening presynaptic action potentials, indicating that voltage-gated potassium channels in the nerve terminal play a regulatory role in transmitter release (Katz and Miledi, 1969; Augustine, 1990). Voltage-gated potassium currents in the nerve terminal can also mediate synaptic modulation by various signal molecules and drugs (Miura et al., 1992; Lupardus et al., 2000; Daniel and Crepel, 2001; Ishikawa and Takahashi, 2001; Lambe and Aghajanian, 2001). Although $>100$ different subunits of potassium channels have been identified (Coetzee et al., 1999), the functional roles of presynaptic potassium channel subtypes in regulating transmitter release remain essentially unknown.

Distinct types of potassium currents have been documented at the peripheral and central nerve terminals (Bielefeldt et al., 1992; Sakaba et al., 1997; Sun et al., 1999; Southan and Robertson, 2000). At the cerebellar basket cell nerve terminal, for example, potassium currents can be attenuated by dendrotoxin (DTX) or

\footnotetext{
Received July 11, 2003; revised Sept. 9, 2003; accepted Sept. 22, 2003.

This study was supported by a Grant-in-Aid for Specially Promoted Research from the Ministry of Education, Culture, Sports, Science and Technology. We thank lan Forsythe and Tetsuhiro Tsujimoto for comments on this manuscript and Peter Strong and Maria Garcia for advice on $\mathrm{K}$ channel blockers.

Correspondence should be addressed to Tomoyuki Takahashi, Department of Neurophysiology, University of Tokyo Graduate School of Medicine, 7-3-1 Hongo, Bunkyo-ku, Tokyo 113-0033, Japan. E-mail: ttakahas-tky@umin.ac.jp.

W.-B. Li's present address: Department of Pathophysiology, Institute of Basic Medicine, Hebei Medical University, Shijiazhuang 050017, People's Republic of China.

Copyright $\odot 2003$ Society for Neuroscience $\quad$ 0270-6474/03/2310445-09\$15.00/0
}

TEA, suggesting the presence of Kv1 and Kv3 channels in the terminal (Southan and Robertson, 2000); however, neither DTX nor TEA increases action potential-evoked $\mathrm{Ca}^{2+}$ influx into the nerve terminal, implying that they may not play a direct regulatory role in evoked transmitter release (Tan and Llano, 1999; Southan and Robertson, 2000). To clarify the role of a presynaptic potassium channel subtype in synaptic transmission, it is desirable to test the effect of type-specific blockers on both presynaptic potassium currents and postsynaptic responses at the same synapse. The calyx of Held seems ideal for this purpose because simultaneous presynaptic and postsynaptic whole-cell recordings are possible (Borst et al., 1995; Takahashi et al., 1996), and its large structure is also advantageous for immunocytochemical examination of presynaptic proteins (Kajikawa et al., 2001; Saitoh et al., 2001). Our results suggest that Kv3 and Kv1 channels play distinct roles in the regulation of transmitter release at this mammalian fast synapse.

\section{Materials and Methods}

Electrophysiology. All experiments were performed in accordance with the guidelines of the Physiological Society of Japan. Transverse brainstem slices (150-200 $\mu \mathrm{m}$ thick) containing the medial nucleus of the trapezoid body (MNTB) region were prepared from Wistar rats, 13-15 d old, killed by decapitation under halothane anesthesia (Forsythe and BarnesDavies, 1993). MNTB principal cells and calyces were visually identified using a $60 \times$ water immersion lens (Olympus Optical, Tokyo, Japan) attached to an upright microscope (Axioskop; Zeiss, Oberkochen, Germany). Each slice was superfused with an artificial CSF (aCSF) contain- 
A
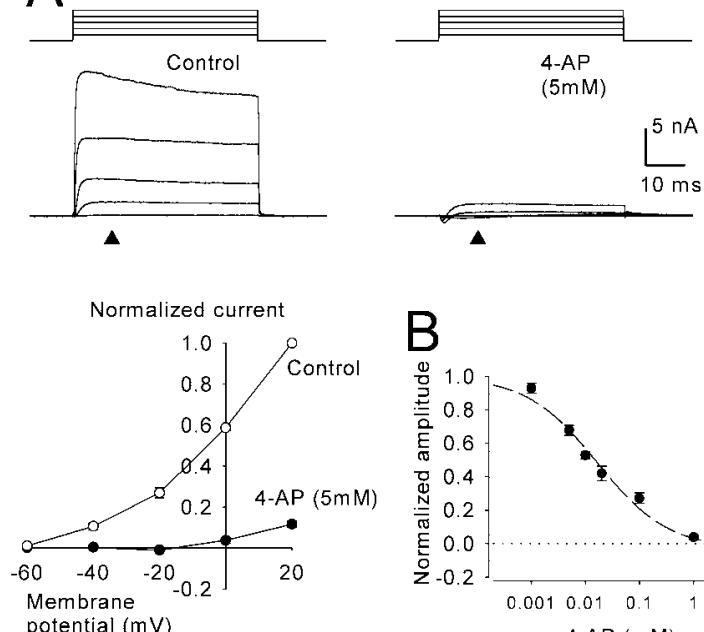

potential $(\mathrm{mV})$

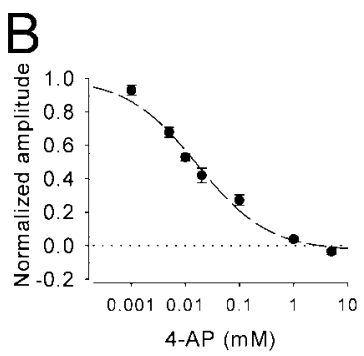

Figure 1. 4-AP blocks presynaptic potassium currents. $A$, Top, $I_{\mathrm{PK}}$ evoked by command pulses from $-80 \mathrm{mV}$ to various potentials by $20 \mathrm{mV}$ steps (from -60 to $+20 \mathrm{mV}$, top) before (left) and during (right) 4-AP (5 mM) application in the presence of TTX. Bottom, The currentvoltage $(I-\bigvee)$ relationships of $I_{\mathrm{PK}}$ before and during 4-AP application $(n=6)$. Current amplitudes were measured at $10 \mathrm{msec}$ from the onset of a command pulse (arrowheads). $B$, Concentration-response relationship for the inhibitory effect of 4-AP on $/$ PK (evoked by depolarization to $-20 \mathrm{mV}$ ). 4-AP was applied incrementally from low to high concentrations at three to four calyces. A curve fitted to data points was drawn according to the equation $y=1-$ (maximal inhibition $) /\left[1+\left(\mathrm{IC}_{50} / x\right)^{n}\right]$, where the maximal inhibition was $1.03, \mathrm{IC}_{50}$ was $17 \mu \mathrm{m}$, and the apparent Hill coefficient $(n)$ was 0.66 . Ordinate indicates the amplitude of $/{ }_{\mathrm{PK}}$ and normalized to control before 4-AP application. Error bars indicate SEMs.

ing (in mu): $125 \mathrm{NaCl}, 2.5 \mathrm{KCl}, 26 \mathrm{NaHCO}_{3}, 10$ glucose, $1.25 \mathrm{NaH}_{2} \mathrm{PO}_{4}$, $2 \mathrm{CaCl}_{2}, 1 \mathrm{MgCl}_{2}, 3$ myo-inositol, 2 sodium pyruvate, 0.5 ascorbic acid, and 4 lactic acid, pH 7.4 with $95 \% \mathrm{O}_{2}$ and $5 \% \mathrm{CO}_{2}$. For recording EPSCs, the superfusate routinely contained bicuculline methiodide $(10 \mu \mathrm{M}$; Sigma, St. Louis, MO) and strychnine hydrochloride ( $0.5 \mu \mathrm{M}$; Sigma) to block inhibitory synaptic responses. The patch pipette solution for postsynaptic recording contained (in $\mathrm{mm}$ ): $110 \mathrm{CsF}, 30 \mathrm{CsCl}, 10 \mathrm{HEPES}$, 5 EGTA, and $1 \mathrm{MgCl}_{2}, \mathrm{pH}$ adjusted to 7.3 with $\mathrm{CsOH}, 290-300 \mathrm{mOsm}$. $\mathrm{N}$-(2,6-diethylphenylcarbamoylmethyl)-triethyl-ammonium chloride (QX314; $5 \mathrm{~mm}$ ) was also included in the pipette solution to block action potential generation. The pipette solution for recording potassium currents from calyceal terminals or from outside-out patches contained (in $\mathrm{mm}$ ): 97.5 potassium gluconate, $32.5 \mathrm{KCl}, 10 \mathrm{HEPES}, 5 \mathrm{EGTA}, 1 \mathrm{MgCl}_{2}$, $12 \mathrm{Na}_{2}$ phosphocreatine, 2 ATP-Mg, and $0.5 \mathrm{GTP}$, pH adjusted to 7.3 with $\mathrm{KOH}, 290-300 \mathrm{mOsm}$. In experiments shown in Figure 2, the EGTA concentration was decreased to $0.2 \mathrm{~mm}$. In experiments shown in Figure 4, potassium gluconate $(97.5 \mathrm{~mm})$ was replaced by equimolar $\mathrm{N}$-methyl-D-glucamine gluconate to improve the voltage-clamp condition. Tetrodotoxin (TTX; $1 \mu \mathrm{M}$ ) (Wako, Osaka, Japan) was included in the aCSF during the whole-cell recording of potassium currents. For simultaneous presynaptic and postsynaptic whole-cell recordings, potassium glutamate $(10 \mathrm{~mm})$ was added to the presynaptic pipette solution by replacing equimolar potassium gluconate, and the EGTA concentration was lowered to $0.5 \mathrm{~mm}$. Drugs were bath-applied by perfusion at $1.5-2$ $\mathrm{ml} / \mathrm{min}$. TEA and 4-AP were purchased from Nacalai (Kyoto, Japan) and Wako, respectively. Synthetic peptide toxins, iberiotoxin (IbTX), margatoxin (MgTX), DTX-I, and tityustoxin-K $\alpha$ (TsTX) were purchased from the Peptide Institute (Osaka, Japan).

EPSCs were evoked at $0.1 \mathrm{~Hz}$ by extracellular stimulation of the presynaptic trapezoid fibers using a tungsten bipolar electrode positioned half-way between the midline and the MNTB, or by presynaptic action potentials elicited directly by a whole-cell pipette under current-clamp mode. Postsynaptic MNTB principal cells were voltage-clamped at the holding potential of $-70 \mathrm{mV}$. EPSCs derived from the calyx of Held synapse were identified as those evoked in an all-or-none manner for graded stimulus intensity and having amplitudes larger than $1 \mathrm{nA}$ at -70
A

(i) $4-\mathrm{AP}$

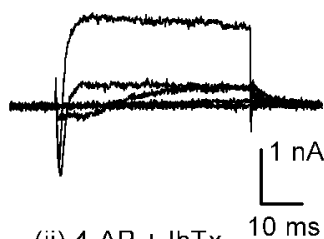

(ii) $4-\mathrm{AP}+\mathrm{IbTx}$

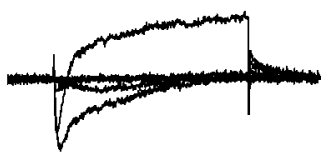

(iii) $4-\mathrm{AP}+\mathrm{IbTx}+\mathrm{Cd}^{2+}$

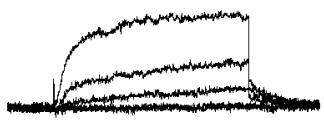

(i) - (ii)

IbTX-sensitive current

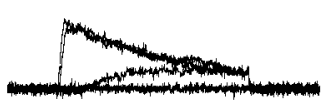

(ii) - (iii)

$\mathrm{Cd}^{2+}$-sensitive current
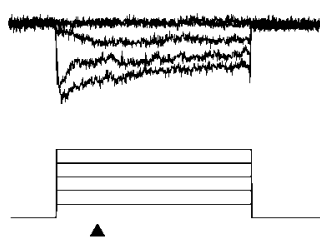

B

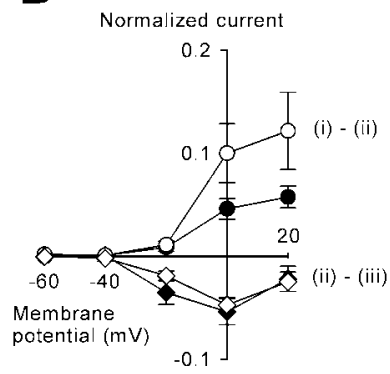

C
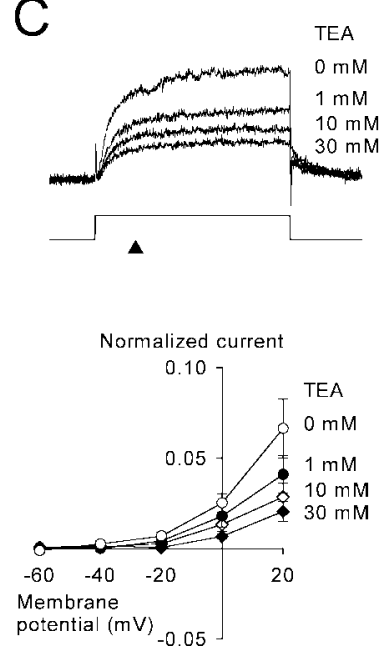

Figure 2. Inward and outward currents remaining after 4-AP application. A, Currents evoked by the command pulses (bottom) in the presence of 4-AP ( $5 \mathrm{~mm}$ ) (i), 4-AP and IbTX (200 nM) (ii), and 4-AP, IbTX, and $\mathrm{CdCl}_{2}(100 \mu \mathrm{m})$ (iii). The IbTX-sensitive outward currents (i-ii) and the $\mathrm{Cd}^{2+}$-sensitive inward $\mathrm{Ca}^{2+}$ currents (ii-iii) were obtained by subtraction. EGTA concentration in the presynaptic pipette solution was $0.2 \mathrm{~mm}$ in these experiments. $B$, The $I-V$ relationships of IbTX-sensitive currents (circles) and $\mathrm{Ca}^{2+}$ currents (diamonds) with $0.2 \mathrm{~mm}$ (open symbols; $n=5$ ) or $5 \mathrm{~mm}$ (filled symbols; $n=6$ ) EGTA in presynaptic pipettes. The current amplitudes are normalized to the total $I_{\text {PK }}$ amplitude at $+20 \mathrm{mV}$. C, The effect of TEA $(1,10,30$ $\mathrm{mm}$ ) on the slow outward currents (iii) remaining in the presence of 4-AP, IbTX, and $\mathrm{Cd}^{2+}$. Sample traces (superimposed) are evoked by a depolarizing pulse to $+20 \mathrm{mV}$. Data points in the $I-V$ relationships are obtained from four experiments.

$\mathrm{mV}$ (Forsythe and Barnes-Davies, 1993). For recording $I_{\mathrm{PK}}$, calyces or outside-out patches excised from calyceal terminals were voltageclamped at a holding potential of $-80 \mathrm{mV}$, and depolarizing voltage steps were applied every $15 \mathrm{sec}$ in the presence of TTX (Forsythe, 1994). The access resistance of postsynaptic recordings was 3-15 M $\Omega$ (electrode resistance, $1.5-4 \mathrm{M} \Omega$ ) and compensated by $80 \%$. The presynaptic access resistance was 6-18 $\mathrm{M} \Omega$ (electrode resistance, 4-6 M $\Omega$ ) and compensated by $60-80 \%$. Leak currents in whole-cell recordings were subtracted by the scaled pulse $(\mathrm{P} / 8)$ protocol. Current recordings with a single pipette were made using a patch-clamp amplifier (Axopatch-1D or Axopatch-200B; Axon Instruments, Foster City, CA). Simultaneous presynaptic and postsynaptic recordings were made using a MultiClamp 700A amplifier (Axon Instruments) equipped with a voltage follower having high input impedance. In conventional patch-clamp amplifiers, in current-clamp mode, action potentials can be distorted in shape or associated with artificial potential changes (Magistretti et al., 1996). The records were low-pass filtered at $5-6 \mathrm{kHz}$ and digitized at $20-50 \mathrm{kHz}$ by an analog-to-digital converter (Digidata 1200A or Digidata 1320A) with 


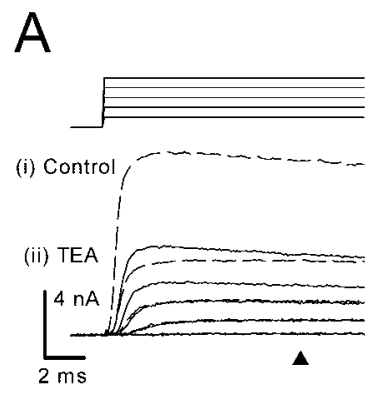

B
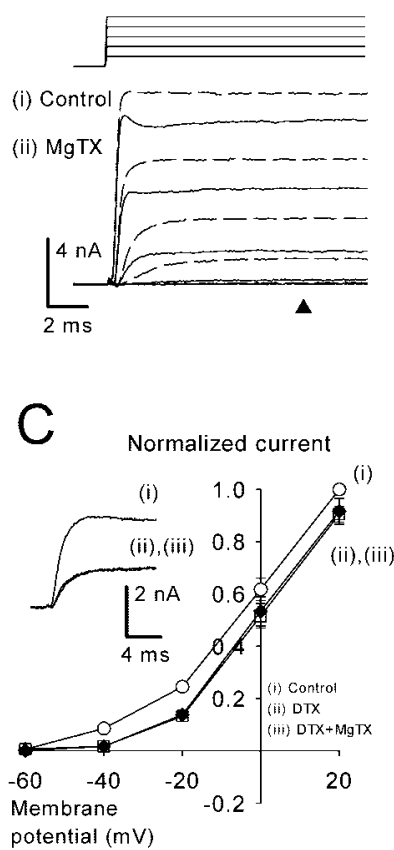

$2 \mathrm{hr}, 15 \%$ for $2 \mathrm{hr}$, and 20\% for overnight. Transverse slices $(30 \mu \mathrm{m}$ in thickness) were cut using a cryostat (CM3050; Leica, Nussloch, Germany) at -21 or $-22^{\circ} \mathrm{C}$. The sections were then processed for immunocytochemistry as follows: (1) blocking and permeablization in PBS containing $4 \%$ skim milk and $0.3 \%(\mathrm{w} / \mathrm{v})$ Triton X-100 overnight at $4^{\circ} \mathrm{C},(2)$ application of primary antibodies in PBS containing $1 \%(\mathrm{w} / \mathrm{v})$ bovine serum albumin and $0.02 \%$ Triton X-100 for $2 \mathrm{~d}$ at $4^{\circ} \mathrm{C}$, (3) application of secondary antibodies in the same buffer as primary antibodies for $2 \mathrm{~d}$ at $4^{\circ} \mathrm{C}$, and (4) mounting with ProLong antifade kit (Molecular Probes, Eugene, OR). Kv channels were detected with mouse monoclonal antiKv1.1, anti-Kv1.2, and anti-Kv1.6 (Upstate Biotechnology, Lake Placid, NY; diluted 1:100), rabbit polyclonal anti-Kv1.3, anti-Kv1.5, anti-Kv2.1, anti-Kv3.2, and anti-Kv3.4 (Alomone, Jerusalem, Israel; diluted 1:100), or rabbit polyclonal anti-Kv1.4 and anti-Kv3.1b antibodies (Chemicon, Temecula, CA; diluted 1:200). The specificity of the antibodies was confirmed by Western blot analysis using a crude sample of brain homogenate. To define presynaptic terminals, we used rabbit anti-synaptophysin antibody (Zymed, South San Francisco, CA; diluted 1:100) or mouse anti-synaptophysin antibody (Chemicon; diluted 1:200). To visualize the $\mathrm{Kv}$ channels and synaptophysin, we used the goat secondary antibodies conjugated with Alexa fluor 488 and Alexa fluor 568 (Molecular Probes; diluted 1:200), respectively. The specificities of Kv channel primary antibodies were evaluated by preabsorption with corresponding antigens (Alomone or Chemicon) for $1 \mathrm{hr}$ at $30^{\circ} \mathrm{C}$. Stained sections were viewed with a $100 \times$ oil-immersion objective (numerical aperture 1.35) using a confocal laser scanning microscope (Fluoview FV300; Olympus Optical). Emission wavelengths were $510-530 \mathrm{~nm}$ (for green) and $>610 \mathrm{~nm}$ (for red). All of the immunocytochemical procedures were performed at room temperature $\left(22-27^{\circ} \mathrm{C}\right)$ unless noted otherwise.

\section{Results}

\section{4-AP-sensitive and -insensitive potassium currents at the calyx of Held terminal}

In whole-cell voltage-clamp recording from calyceal nerve terminals, sustained outward currents were evoked by voltage steps from a holding potential of $-80 \mathrm{mV}$ in the presence of TTX (Fig. $1 A)$. These currents were detectable on depolarization positive to $-40 \mathrm{mV}$, and their amplitude increased steeply for larger voltage steps. These currents had fast activation kinetics with a $10-90 \%$ rise time of $0.68 \pm 0.15 \mathrm{msec}$ (at $+20 \mathrm{mV}, n=6$ ). The potassium channel blocker 4-AP at $5 \mathrm{~mm}$ blocked most of these currents (Fig. 1A), as reported previously (Forsythe, 1994; Ishikawa and Takahashi, 2001). Although 4-AP can undergo use-dependent unblock (Campbell et al., 1993), this is unlikely to contribute significantly to our measurements made $10 \mathrm{msec}$ after the onset of depolarization. The effect of 4-AP was concentration dependent, and the $\mathrm{IC}_{50}$ estimated for the currents evoked by a voltage step to $-20 \mathrm{mV}$ was $17 \mu \mathrm{M}$ (Fig. $1 B$ ).

After most of $I_{\mathrm{PK}}$ were blocked by 4-AP ( $\left.5 \mathrm{mM}\right)$, small currents having inward and outward components remained (Fig. 2A). IbTX (100-200 nM), a large-conductance $\mathrm{Ca}^{2+}$-activated potassium (BK) channel-specific blocker, attenuated these outward currents. The IbTX-sensitive currents obtained by subtraction had a mean rise time (10-90\%) of $2.2 \pm 0.4 \mathrm{msec}(n=5$, at +20 $\mathrm{mV}$ with $0.2 \mathrm{~mm}$ EGTA in the pipette) (Fig. $2 A$ ) and comprised $12 \pm 4 \%(n=5)$ of total $I_{\mathrm{PK}}$ at $+20 \mathrm{mV}$ (Fig. $\left.2 B\right)$. When the EGTA concentration in the pipette was higher $(5 \mathrm{~mm})$, the IbTXsensitive current was smaller $(7 \pm 2 \% ; n=6)$ (Fig. $2 B$ ) and slower (10-90\% rise time; $3.9 \pm 0.7 \mathrm{msec} ; n=6)$, being consistent with the $\mathrm{Ca}^{2+}$-activated nature of the current. In the presence of 4-AP and IbTX, inward and outward currents still remained. $\mathrm{Cd}^{2+}(100 \mu \mathrm{M})$ abolished these inward currents, indicating that they were high-voltage-activated (HVA) $\mathrm{Ca}^{2+}$ currents. The maximal amplitude of $\mathrm{Ca}^{2+}$ currents (at $0 \mathrm{mV}$ ) corresponded to $8 \pm 1 \%(n=5)$ of total $I_{\mathrm{PK}}$ at the same voltage (Fig. $2 \mathrm{~B}$ ). In the presence of 4-AP (5 mM), IbTX (100-200 nM), with sucrose of were cryoprotected at $4^{\circ} \mathrm{C}$ in $0.1 \mathrm{M}$ sodium phos $\mathrm{for}$ 
A

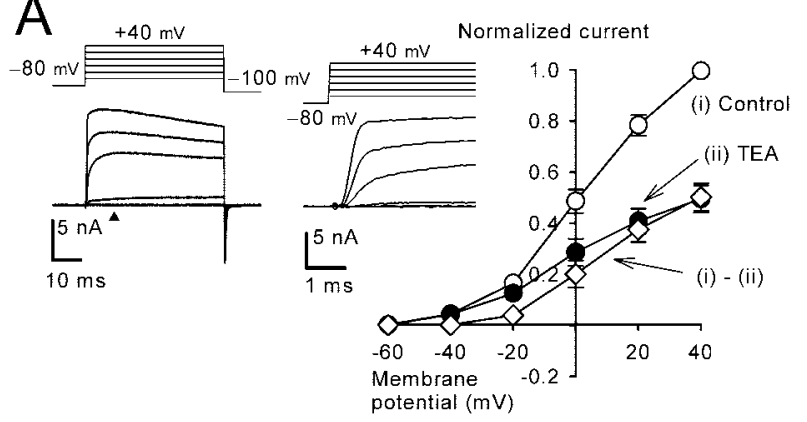

B

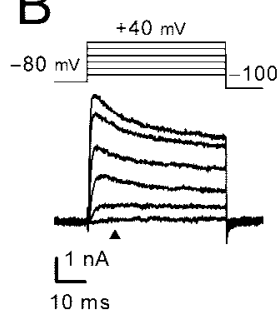

Normalized current

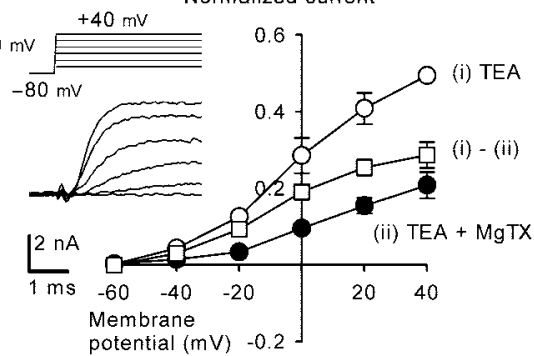

C

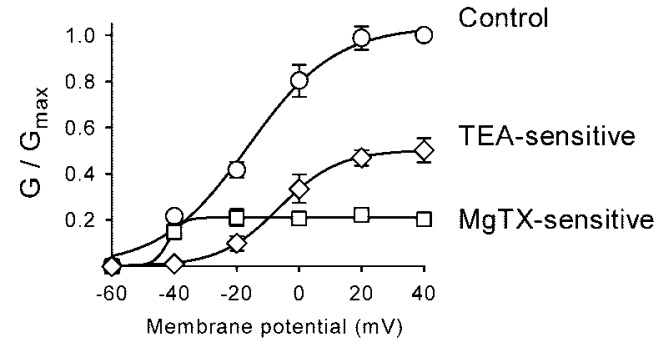

D

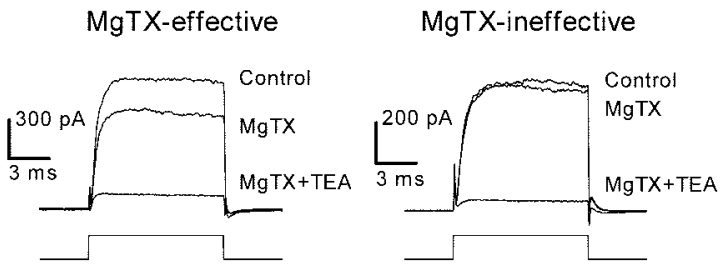

Figure 4. Potassium currents recorded from calyceal whole terminal with reduced intracellular potassium concentration and those recorded from outside-out patches excised from calyces. $A$, $I-V$ relationships before (i) and after (ii) application of TEA (1 mM) and TEA-sensitive difference currents (i-ii) in the presence of C ${ }^{2+}(100 \mu \mathrm{m})$. Sample records of TEA-sensitive currents are shown in the insets at two different time scales. $B, I-V$ relationships before (i) and after (ii) application of MgTX (10 nM) and MgTX-sensitive currents (i-ii) in the presence of TEA (1 mM) and C ${ }^{2+}$ (100 $\mu$ M). Sample records of MgTX-sensitive currents are shown in the insets at two different time scales. C, Whole-terminal chord conductance of TEA- and MgTX-sensitive currents both normalized to that of the total presynaptic potassium conductance (Control). Fitting curves are drawn according to the Boltzmann equation $G=G_{\max } /\left\{1+\exp \left[\left(V-V_{1 / 2}\right) / k\right]\right\}$, where $G_{\text {max }}$ was 1.0 for the total current, 0.50 for the TEA-sensitive current, and 0.21 for the MgTX-sensitive current. The half-activation potential $\left(V_{1 / 2}\right)$ was $-16 \mathrm{mV}$ for the total current, $-6.5 \mathrm{mV}$ for the TEA-sensitive current, and -42 $\mathrm{mV}$ for the MgTX-sensitive current. The apparent slope factor $(k)$ was $14 \mathrm{mV}$ for the total current, $9.6 \mathrm{mV}$ for the TEA-sensitive current, and $2.3 \mathrm{mV}$ for the MgTX-sensitive current. $D$, Potassium currents recorded from outside-out patches excised from calyceal terminals, before and after application of MgTX and addition of TEA (superimposed). MgTX attenuated outward currents in one patch (left) but had no effect in another patch (right). Voltage step was from -80 to $0 \mathrm{mV}$.

and $\mathrm{Cd}^{2+}(100 \mu \mathrm{M})$, slowly activating outward currents were discerned above $-40 \mathrm{mV}$. This component comprised $7 \pm 1 \%$ (with $0.2 \mathrm{~mm}$ EGTA, $n=6$; or $8 \pm 1 \%$ in 5 mM EGTA, $n=5$ ) of total $I_{\mathrm{PK}}$ at $+20 \mathrm{mV}$ (Fig. $2 \mathrm{~A}$, iii). The slow activation kinetics of the outward currents ( $10-90 \%$ rise time; $42 \pm 2 \mathrm{msec}$ at $+20 \mathrm{mV}$; $n=6$ ) suggests that these derive from channels distinct from those comprising the main component of $I_{\mathrm{PK}}$. TEA attenuated this component in a concentration-dependent manner (Fig. 2C). Thus the voltage-dependent potassium currents at the calyx of Held terminal are composed of a 4-AP-sensitive main component and a much smaller 4-AP-insensitive component comprising BK channel currents and an unidentified slowly activating potassium current.

\section{TEA- and MgTX-sensitive potassium currents}

A low concentration of TEA ( $1 \mathrm{mM})$ attenuated $I_{\mathrm{PK}}$ at the membrane potential above $-20 \mathrm{mV}$ (Fig. 3A), suggesting that HVA currents predominate the TEA-sensitive currents. The effect of TEA was reversible after washout (data not shown), and the TEA block was concentration dependent (Fig. 3A). The 4-APinsensitive BK currents were sensitive to TEA, and slowly activating potassium currents were also partially blocked by 1 mM TEA (Fig. 2). Therefore, TEA-sensitive currents are likely to include these 4-AP-insensitive currents as a minor component. The main TEA-sensitive component has an HVA nature, fast activation kinetics (Fig. 4A), and was sensitive to 4-AP, suggesting that it arises from Kv3 channels (Coetzee et al., 1999).

The scorpion peptide MgTX blocks Kv1.3 channels at picomolar concentrations and Kv1.6 channels at nanomolar concen- trations (Garcia-Calvo et al., 1993). MgTX may also block Kv1.2 channels given its binding activity to Kv1.2 in rat brain synaptosomes (Knaus et al., 1995). MgTX (10 nM) attenuated $I_{\mathrm{PK}}$ (Fig. $3 B$ ), with its effect not reversible for at least $30 \mathrm{~min}$ after washout. The MgTX-sensitive currents had a low-voltage-activated (LVA) nature, with a clear activation at $-40 \mathrm{mV}$. Similar to MgTX, DTX-I (200 nM), which blocks Kv1.1, 1.2, and 1.6 (Harvey, 1997), also attenuated $I_{\mathrm{PK}}$ (Fig. 3C). In addition, TsTX (100 nM), which specifically blocks Kv1.2 (Hopkins, 1998), attenuated LVA $I_{\mathrm{PK}}$, as reported recently (Dodson et al., 2003). In the presence of DTX-I or TsTX, MgTX no longer affected $I_{\mathrm{PK}}$. Because all of these toxins can block Kv1.2 channels, these results suggest that the toxinsensitive LVA potassium currents may derive from either all homomeric Kv1.2 channels or heteromeric channels containing Kv1.2. Given that Kv1 channels are generally sensitive to 4-AP (Coetzee et al., 1999), these channels are likely to comprise the 4-AP-sensitive main component of $I_{\mathrm{PK}}$, particularly at the low voltage range.

\section{Contribution of $\mathrm{Kv} 3$ and $\mathrm{Kv} 1$ components to the presynaptic potassium currents}

We next examined the relative contribution of Kv3 and Kv1 channels to total $I_{\mathrm{PK}}$ after improving the voltage-clamp condition by reducing the potassium concentration in the presynaptic pipette by $75 \%$ (see Materials and Methods). We first applied $\mathrm{Cd}^{2+}$ $(100 \mu \mathrm{M})$ and blocked both $\mathrm{Ca}^{2+}$ channels and $\mathrm{Ca}^{2+}$-activated potassium channels. In the presence of $\mathrm{Cd}^{2+}$, we then applied TEA (1 mM) (Fig. 4A) followed by MgTX (10 nM) in addition to TEA (Fig. $4 B$ ). The TEA-sensitive current, which was obtained by 
subtracting currents in the presence of TEA from control (Fig. $4 A)$, had a fast rise time $(10-90 \% ; 0.54 \pm 0.05 \mathrm{msec}$ at $+20 \mathrm{mV}$; $n=5$ ) compared with the MgTX-sensitive current (Fig. $4 B$ ) $(10-90 \%$ rise time; $1.2 \pm 0.1 \mathrm{msec}$ at $+20 \mathrm{mV} ; n=4)$. The activation curve of the TEA-sensitive current (deduced from chord conductance) had an HVA nature with a maximal conductance corresponding to $50 \pm 5 \%$ of total $I_{\mathrm{PK}}$ conductance (at +40 $\mathrm{mV} ; n=5$ ) (Fig. 4C). In contrast, the MgTX-sensitive current had an LVA nature, with its maximal conductance corresponding to $20 \pm 5 \%(n=4)$ of total $I_{\mathrm{PK}}$ conductance (Fig. $\left.4 C\right)$. To examine whether there is a current component sensitive to both TEA and MgTX, in another set of experiments we reversed the order by applying MgTX first, followed by application of TEA and MgTX. The TEA-sensitive conductance measured in the presence of MgTX was $51 \pm 9 \%$ (at $+40 \mathrm{mV} ; n=4$ ) of total $I_{\mathrm{PK}}$ conductance, which was similar to that obtained in the absence of MgTX $(50 \pm 5 \%)$, suggesting that there is little overlap between the TEA- and the MgTX-sensitive currents.

Although the TEA-sensitive conductance contains a small portion of 4-AP-insensitive, slow-activating currents (Fig. 2C), the above results suggest that $\sim 50 \%$ of $I_{\mathrm{PK}}$ derives from $\mathrm{Kv} 3$ channels. In outside-out patches excised from the calyceal terminals, TEA attenuated outward currents in all six patches examined (Fig. $4 \mathrm{D}$ ) on average by $83 \pm 3 \%$ (at $0 \mathrm{mV}$ ), suggesting that $\mathrm{Kv} 3$ is the main potassium channel expressed on the nonsynaptic surface of the calyces. In this regard, it has been reported recently that Kv3.1b-specific immunogold particles are localized on the nonsynaptic side of the calyx of Held terminal (Elezgarai et al., 2003). MgTX (10 nM) attenuated outward currents in three patches by $27 \pm 4 \%$ on average but had no effect in four other patches (Fig. $4 D$ ), suggesting that the density of Kv1 channels at the calyceal terminal is low. Given the substantial contribution of the MgTX-sensitive current to $I_{\mathrm{PK}}$ (Fig. $4 B$ ), Kv1 channels may also be expressed at other regions, such as the presynaptic axon, as suggested recently (Dodson et al., 2003).

\section{Immunocytochemical examination of potassium channel subunits at the calyx of Held terminal}

Using subunit-specific antibodies, we examined which subtypes of Kv1 and Kv3 channels were expressed at the calyceal terminal. To distinguish Kv channel immunofluorescence from nonspecific background signals, the primary antibodies were preabsorbed with corresponding antigens (Fig. 5, bottom panels). Fluorescence intensity was measured using densitometry after background subtraction. Presynaptically localized Kv channels were identified by an overlap with synaptophysin immunofluorescence (Fig. 5) (Kajikawa et al., 2001; Saitoh et al., 2001). At the calyx of Held, clear signals were observed for Kv3.1b and Kv 3.4 (Fig. 5). Although less clear, positive signals were also discerned for Kv1.1, Kv1.2, and Kv1.3. Signals for Kv1.4, Kv1.5, Kv1.6, and $\mathrm{Kv} 3.2$ were marginal, and there was no Kv2.1 immunoreactivity at the terminal. All $\mathrm{Kv}$ channels examined were more or less expressed in the somatic region of MNTB neurons. Although the fluorescence intensity depends partially on the quality of antibodies, these results provide a rough idea regarding $\mathrm{Kv}$ channel subtypes expressed at the calyx of Held synapse.

\section{Effects of potassium channel blockers on EPSCs}

We examined whether Kv1 and Kv3 channels play regulatory roles in synaptic transmission by testing the effect of potassium channel blockers on EPSCs. As reported previously (Ishikawa and Takahashi, 2001), 4-AP increased the EPSC amplitude in a

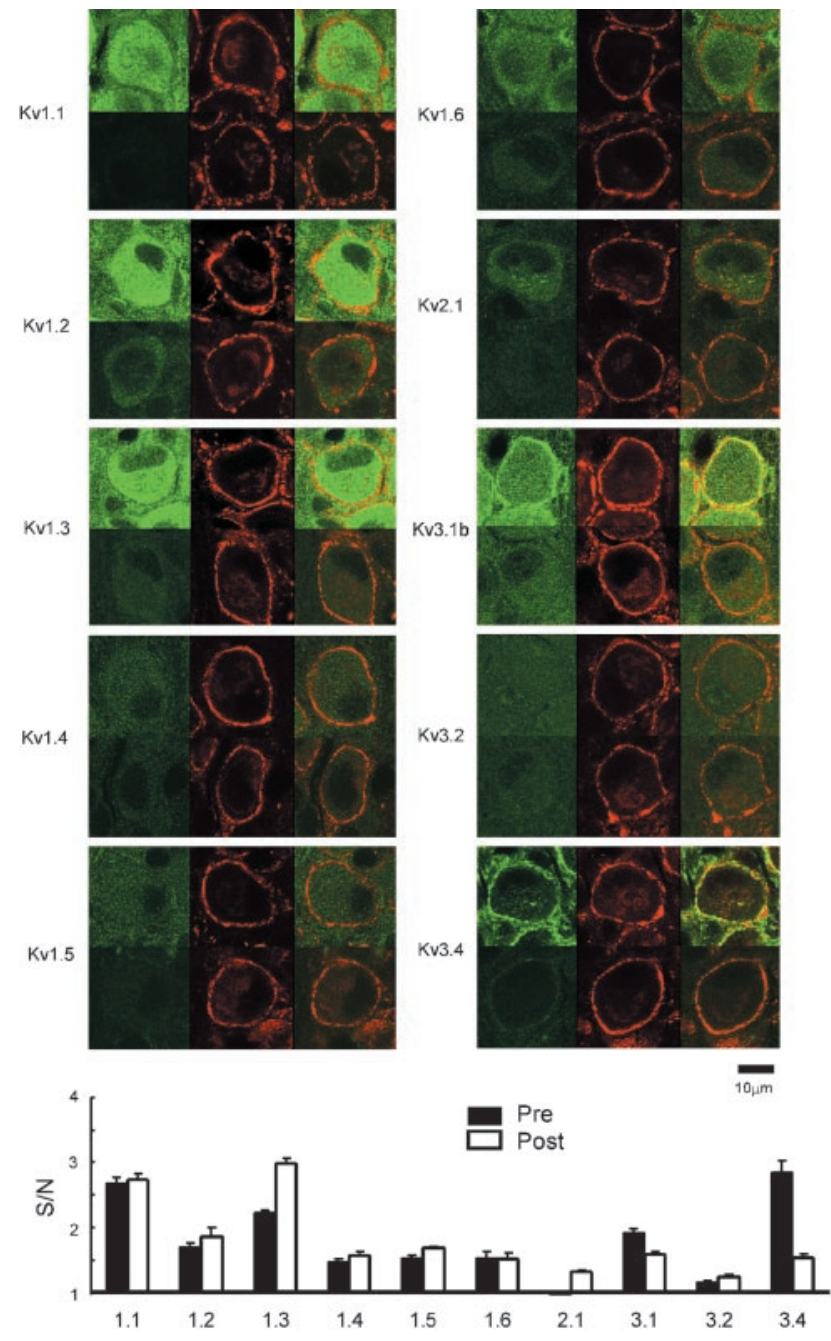

Figure 5. Immunoreactivity of Kv channels at the calyx of Held. Each bottom panel shows the background after absorbing the primary antibodies of Kv channels with corresponding antigens. Ordinate $(\mathrm{S} / \mathrm{N})$ in the bar graphs (bottom) indicates the backgroundsubtracted signal intensity divided by the background intensity. Immunofluorescence signals of Kv channels that overlapped with synaptophysin signals were taken as presynaptic signals, and their averaged intensities were determined by densitometric measurements (filled columns). Intensities of somatic signals (open columns) were estimated from signals of Kv channels surrounded by synaptophysin signals excluding nucleus. Each data derived from 16 to 24 cells in three slices.

concentration-dependent manner (Fig. 6A,B), with a nearly threefold increase at $100 \mu \mathrm{M}$ (potentiation by $188 \pm 35 \% ; n=9$ ). Above $100 \mu \mathrm{M}, 4$-AP caused presynaptic action potential firing (data not shown), which induced EPSC bursts and synaptic depression (Fig. 6A). Similar to 4-AP, TEA potentiated EPSCs in a concentration-dependent manner (Fig. 6C,D), with a maximal potentiation being $99 \pm 7 \%$ at $1-10 \mathrm{mM}(n=16)$. Unlike $4-\mathrm{AP}$, TEA did not cause a burst of EPSCs even at its maximal concentration. Despite the presence of IbTX-sensitive currents in the nerve terminal (Fig. 2), IbTX (200-300 nM) had no effect on EPSCs (data not shown).

To study the mechanism underlying synaptic potentiation by TEA, we made simultaneous presynaptic and postsynaptic recordings and evoked EPSCs by presynaptic action potentials (elicited using $1 \mathrm{msec}$ depolarizing pulses) (Fig. 7). After TEA application (1 $\mathrm{mm})$, presynaptic action potentials became 
broader, with half-width increasing by $68 \pm 3 \%(n=5 ; p<0.001)$, and became larger in peak amplitude (by $10 \pm 2 \%$; $n=$ $5 ; p<0.05)$. There was a sublinear relationship between the half-width of presynaptic action potential and the amplitude of EPSCs (Fig. 7). TEA had no effect on the presynaptic resting potential (change by $2 \pm 1 \% ; n=5)$.

We next examined the effect of MgTX in simultaneous presynaptic and postsynaptic recordings. In contrast to TEA, MgTX had no effect on the amplitude or waveform of presynaptic action potentials or on the EPSC amplitude in simultaneous recordings (change by $3 \pm 3 \% ; n=3$ ) (Fig. $8 A$ ), as well as in single postsynaptic recordings (by $3 \pm 2 \% ; n=4$ ). MgTX also had no effect on the presynaptic resting potential $(0 \pm 1 \% ; n=5)$. The lack of effect of MgTX on EPSCs evoked by a presynaptic action potential might arise from the relatively slow activation kinetics of the MgTX-sensitive channel (10-90\% rise time $1.2 \pm 0.1 \mathrm{msec}$ ) (Fig. 4). Because presynaptic action potentials last no longer than $1 \mathrm{msec}$, the activation time of the MgTX-sensitive channels may not be fast enough to affect presynaptic action potentials. MgTX might then have prolonged EPSCs if presynaptic action potentials had been broadened. This was tested by broadening presynaptic action potentials by TEA (1 mM) (Fig. 8B). MgTX, applied in the presence of TEA, further prolonged presynaptic action potential duration (half-width, by $36 \pm 11 \% ; n=5$ ) and potentiated EPSCs (by $21 \pm 7 \% ; n=5$ ) (Fig. $8 B$ ). MgTX had no effect on the action potential amplitude $(2 \pm 4 \% ; n=$ $5)$. These results suggest that Kv1 channels can regulate transmitter release only when the action potential is broadened.

A prominent effect of MgTX was observed when the nerve terminal was depolarized for a sustained period (Fig. 9). Suprathreshold presynaptic depolarization by a long depolarizing pulse (100 msec) normally evokes a single action potential (Fig. 9A), with the number of action potentials increasing to 3.6 on average ( $n=$ 5 ) by further depolarization (with injection currents up to 240 $\mathrm{pA}$ ). In the presence of MgTX, however, a sustained presynaptic depolarization induced a burst of action potentials associated with a train of EPSCs of decreasing amplitude (Fig. 9A). MgTX significantly lowered the minimal current required for generating action potential (by $49 \pm 5 \% ; n=5 ; p<0.005$ ) (Fig. 9B) and increased the maximum number of action potentials (by $858 \pm$ $362 \% ; n=5)$. In contrast to MgTX, TEA ( $1 \mathrm{~mm}$ ) did not induce a burst of spikes on prolonged depolarization but slightly reduced the minimal current required for spike generation (by $23 \pm 4 \%$; $n=3)$. These results suggest that the MgTX-sensitive LVA channels play a stabilizing role in the nerve terminal.

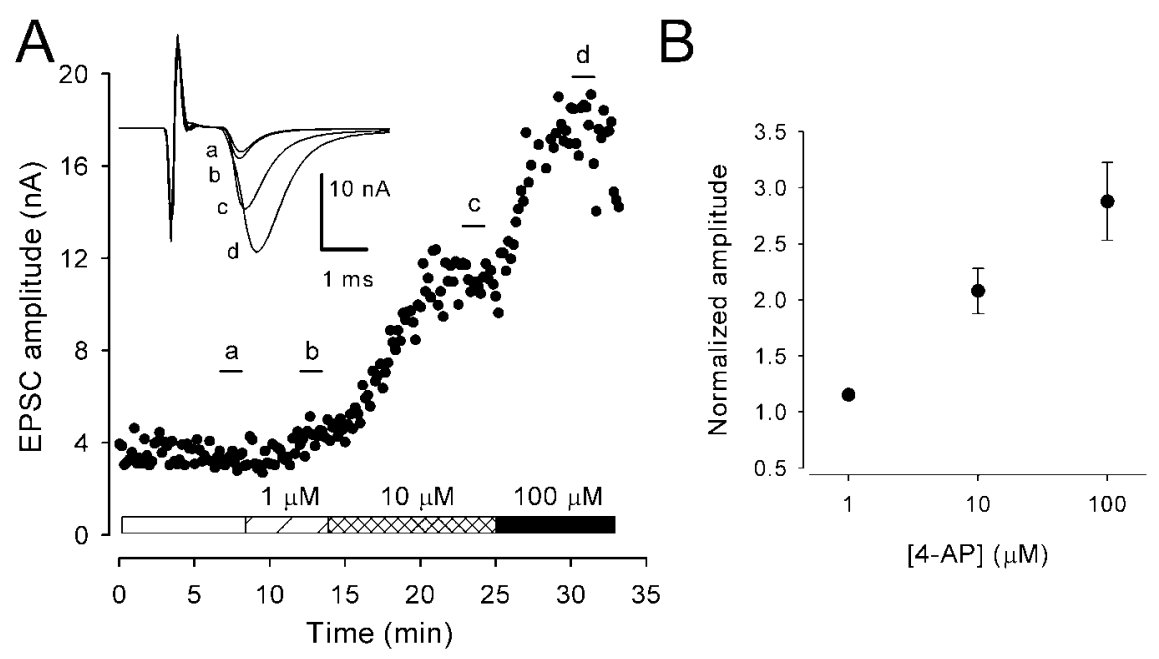

Figure 6. The facilitatory effects of 4-AP and TEA on EPSCS. EPSCS evoked by fiber stimulation were recorded from MNTB neurons at the holding potential of $-70 \mathrm{mV}$. $A$, Three concentrations of 4-AP $(1,10,100 \mu \mathrm{m})$ were applied incrementally. Note applications of $1 \mu \mathrm{M}$ (b), $10 \mu \mathrm{M}$ (c), and $100 \mu \mathrm{M}$ (d) 4-AP (superimposed). B, The concentration-response relationship for the rerds are averaged EPSCs during application of $0.01 \mathrm{~mm}(\mathrm{a}), 0.1 \mathrm{~mm}(\mathrm{~b}), 3 \mathrm{~mm}(\mathrm{c})$, and $10 \mathrm{~mm}$ (d) TEA (superimposed). D, The concentration-response relationship for the facilitatory effect of TEA on EPSCs. Data from 6-16 experiments at each concentraequation $y=1+\left(\right.$ maximal increase) $/\left[1+\left(\mathrm{EC}_{50} / \mathrm{x}\right)^{n}\right]$, where the maximal increase was $1.11, \mathrm{EC}_{50}$ was $0.22 \mathrm{~mm}$, and the apparent Hill coefficient $(n)$ was 1.13 .

\section{Discussion}

Identification of potassium channels expressed at the calyx of Held terminal

At the calyx of Held terminal the voltage-dependent potassium currents have two main components. One is the HVA current sensitive to both 4-AP and TEA. The other is the LVA current sensitive to 4-AP, MgTX, DTX-I, and TsTX. In addition, $I_{\mathrm{PK}}$ contained a minor 4-AP-insensitive component including IbTXsensitive currents and slowly activating potassium currents. Pharmacological properties of the 4-AP-sensitive HVA and LVA currents suggest that they derive mainly from the Kv3 and Kv1 channels, respectively (Coetzee et al., 1999). The results of occlusion experiments suggest that most of the LVA channels are homomeric Kv 1.2 channels or heteromeric channels containing 

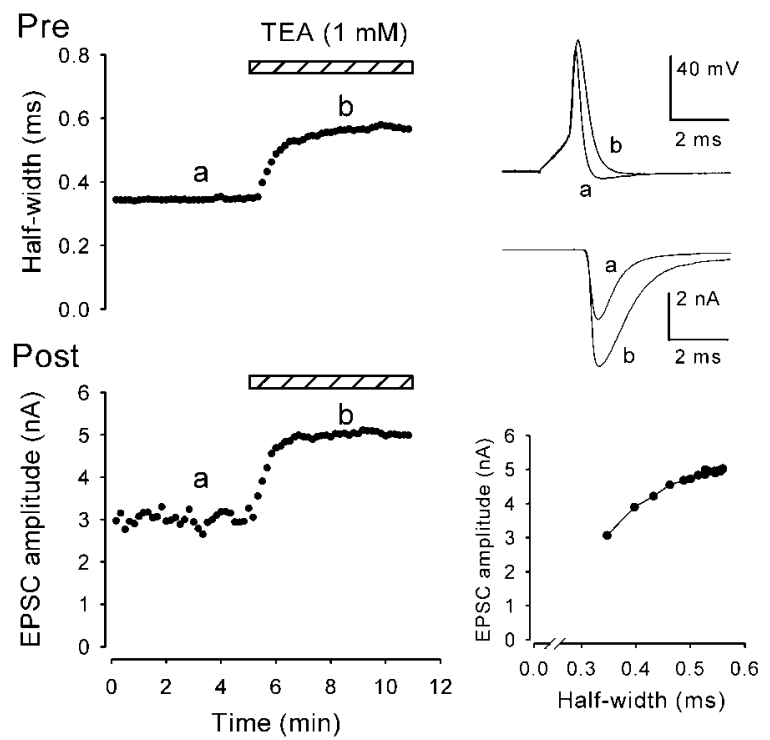

Figure 7. The effect of TEA on presynaptic action potentials and EPSCs in a simultaneous presynaptic and postsynaptic whole-cell recording. Presynaptic action potentials were evoked by a depolarizing pulse of $1 \mathrm{msec}$ in duration. TEA prolonged presynaptic action potential duration and potentiated EPSCS. The half-width of action potential was measured as a duration between $50 \%$ rise time and $50 \%$ decay time (measured from baseline) of action potentials. Sample records are averaged presynaptic action potentials and EPSCs before (a) and during (b) application of TEA (1 mM) (superimposed). Presynaptic resting potential was $-70 \mathrm{mV}$. Right bottom, The relationship between the half-width of presynaptic action potential and the amplitude of EPSC during TEA application.
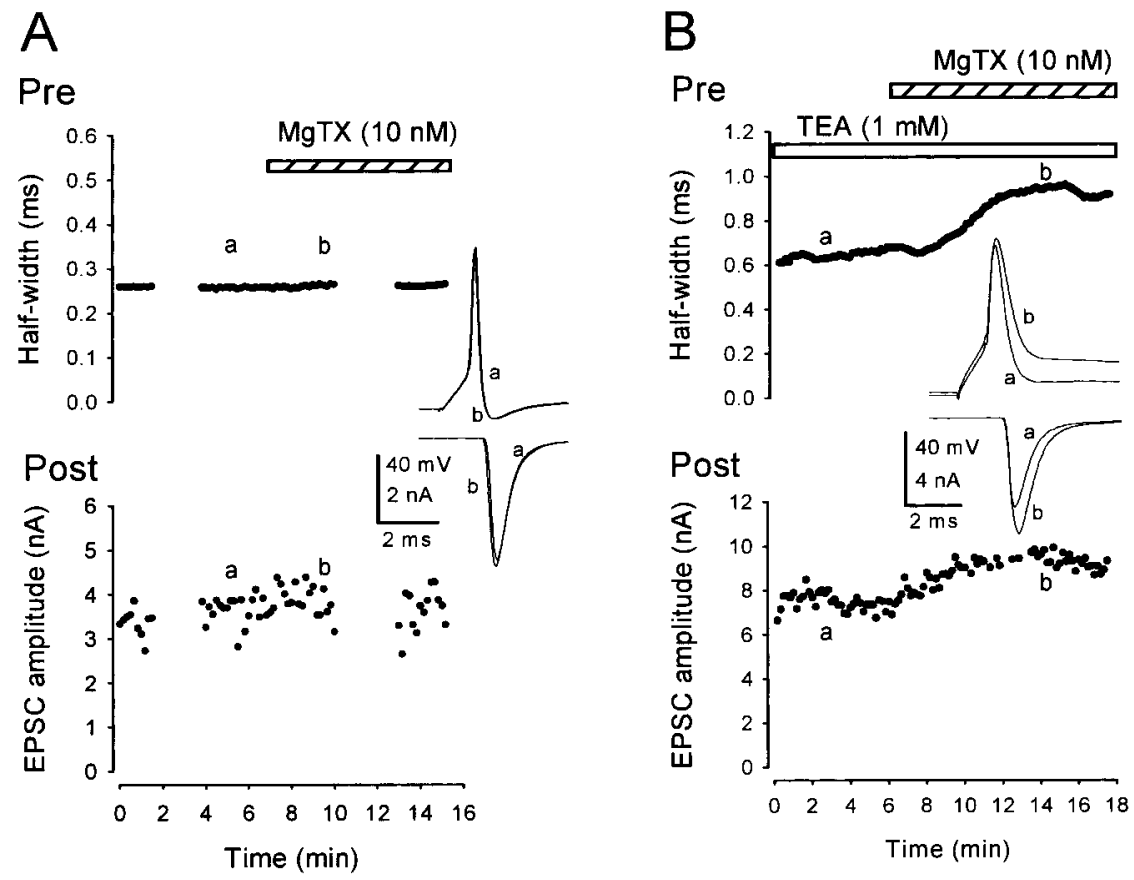

Figure 8. The effect of MgTX on the presynaptic action potential and EPSC. A, MgTX (10 nм) had no effect on presynaptic action potential (sample records on top panel) or EPSCS (bottom panel). Presynaptic resting potential was $-71 \mathrm{mV}$. Gaps in the time plot are caused by experiments for Figure $9 . B, \mathrm{MgTX}(10 \mathrm{~nm})$ applied in the presence of TEA (1 $\mathrm{mm})$ prolonged presynaptic action potential duration and potentiated EPSCS. Presynaptic resting potential was $-78 \mathrm{mV}$. Sample records in $A$ and $B$ are averaged presynaptic action potentials (top panels) and EPSCS (bottom panels) before (a) and during (b) application of MgTX (superimposed).

Kv1.2 subunits. In this regard, it has been suggested recently that Kv1.2 homomers coexist with Kv1.1/1.2 heteromers at the presynaptic site of the calyx of Held synapse (Dodson et al., 2003). Recombinant homomeric Kv1.1 has a moderate TEA sensitivity
$\left(\mathrm{IC}_{50}, 0.3 \mathrm{~mm}\right.$ ), whereas $\mathrm{Kv1.1/1.2}$ heteromer is $>10$ times less sensitive to TEA (Hopkins, 1998). This is consistent with our results indicating little overlap between TEA- and MgTXsensitive currents; however, given multiple Kv1 subunits expressed at the calyceal terminal (Fig. 5), the exact composition of Kv1 channels remains to be determined.

Transcripts for Kv3.1 and 3.3 are expressed in the anterior ventral cochlear nucleus, where the cell body that gives rise to the calyx of Held is located (Weiser et al., 1994; Rudy et al., 1999). Among recombinant channels composed of Kv3 channels, those comprising Kv3.1 have the highest 4-AP sensitivity, with an $\mathrm{IC}_{50}$ of 20-600 $\mu \mathrm{M}$ (Mathie et al., 1998; Coetzee et al., 1999), comparable with that for $I_{\mathrm{PK}}$ recorded from the calyx of Held. Although the presence of Kv3.3 is not testable because of a lack of specific antibody, the calyx terminal showed clear immunoreactivities against Kv3.1 and Kv3.4. Thus these channels may together contribute to the TEA-sensitive HVA currents at the calyx of Held presynaptic terminal.

\section{Identification of potassium channels regulating transmitter release at the calyx of Held synapse}

Bath application of 4-AP or TEA caused a marked potentiation of EPSCs via broadening presynaptic action potentials (Wang and Kaczmarek, 1998; Ishikawa and Takahashi, 2001). In contrast to the calyx of Held in mice (Wang and Kaczmarek, 1998), TEA significantly increased the peak amplitude of presynaptic action potential, suggesting that the activation of $\mathrm{Kv} 3$ channels is fast enough to attenuate the overshoot of presynaptic action potentials in the rat calyceal terminal. MgTX blocked the TEA-insensitive $I_{\mathrm{PK}}$ but had no direct effect on basal synaptic transmission. Although it has been reported that recently TsTX causes aberrant action potentials in presynaptic recordings (Dodson et al., 2003), we never saw MgTX alone causing aberrant presynaptic action potentials or aberrant burst of EPSCs, either in the paired recordings or in single presynaptic or postsynaptic recordings. Only after presynaptic action potentials were broadened by TEA did MgTX further prolong their duration and enhance EPSCs. These results suggest that the activation kinetics of Kv1 channels is too slow to affect presynaptic action potentials in a normal condition. Even in the presence of both MgTX and TEA, no aberrant presynaptic firings were observed. Although Kv1 channels do not normally contribute to action potential duration or maintaining resting potential, they raise the threshold level of current required for presynaptic action potential generation. Thus, during a small but sustained depolarization of nerve terminals, the LVA channels are activated, thereby stabilizing the nerve terminal.

The IbTX-sensitive BK channels are expressed in amphibian motor nerve terminals (Robitaille et al., 1993; Yazejian et al., 2000), in chick autonomic nerve terminals (Sun et al., 1999), in fish retinal bipolar cells (Sakaba et al., 1997), in mammalian pituitary cells (Bielefeldt et al., 1992), and at the calyx of Held terminal of rats (Fig. 2). At the amphibian motor nerve terminal, 

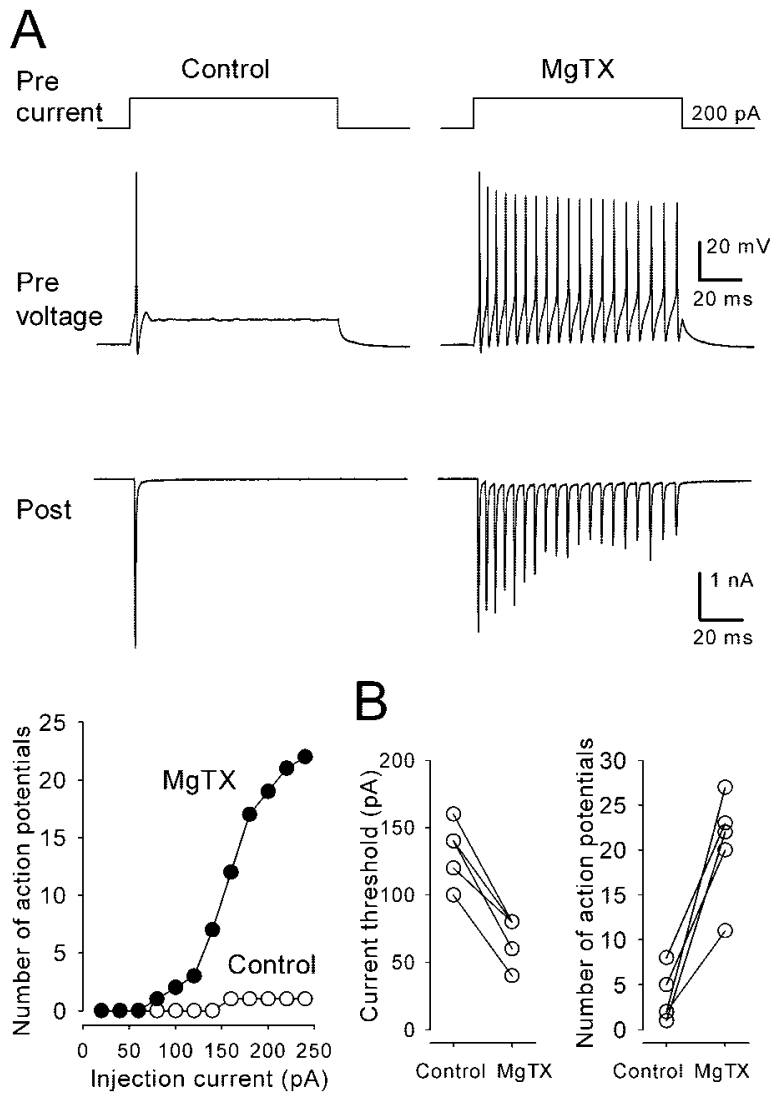

Figure 9. MgTX caused a burst of presynaptic action potentials during sustained depolarization. A, Presynaptic action potentials evoked by a long depolarizing pulse (100 msec) accompanied by EPSCs before (left panel) and during (right panel) application of MgTX (10 nM). Bottom panel, Number of action potentials (ordinate) evoked by depolarizing currents of various intensities (abscissa). Presynaptic resting potential was $-71 \mathrm{mV}$. B, Left panel, The minimal current amplitude to evoke presynaptic action potentials before and during MgTX application. Right panel, The maximum number of action potentials elicited by a depolarizing pulse (100 msec) increased up to $240 \mathrm{pA}$ before and during MgTX application. Data in B derived from three paired recordings and two single presynaptic recordings.

BK channels are activated during $\mathrm{Ca}^{2+}$ entry through nearby voltage-dependent $\mathrm{Ca}^{2+}$ channels, thereby effectively regulating transmitter release (Robitaille et al., 1993). In contrast, at the calyx of Held terminal, the BK channel blocker IbTX had no effect on EPSCs. Because the activation kinetics of presynaptic BK currents $(10-90 \%$ rise time; $2.2 \pm 0.4 \mathrm{msec})$ is even slower than that of the MgTX-sensitive currents (1.2 $\pm 0.1 \mathrm{msec})$, BK channels cannot prolong action potential duration at this mammalian synapse. Similarly, at the hippocampal synapse, IbTX potentiates EPSCs only in the presence of 4-AP (Hu et al., 2001). The faster activation kinetics of $\mathrm{BK}$ channel currents at the amphibian neuromuscular junction (Yazejian et al., 2000) and in retinal bipolar cells (Sakaba et al., 1997) might arise from distinct $\beta$ subunits (Weiger et al., 2000). Thus, in contrast to the amphibian neuromuscular junction, at the mammalian central synapse there is no evidence that $\mathrm{BK}$ channels play a regulatory role in transmitter release.

\section{Physiological roles of presynaptic potassium channels at the calyx of Held synapse}

HVA Kv3 channels with slow inactivation kinetics ensure reproducible shortenings of presynaptic action potentials. As proposed for the postsynaptic MNTB neurons (Brew and Forsythe, 1995), this is essential for the high-fidelity transmission at the calyx of
Held synapse required for the binaural processing of soundsource localization. This contrasts with the pituitary nerve terminals (Jackson et al., 1991) or hippocampal mossy fiber terminal (Geiger and Jonas, 2000), in which fast-inactivating potassium channels broaden action potential duration in an activitydependent manner, thereby facilitating transmitter release.

The LVA Kv1 channels also contribute to the high-fidelity transmission by preventing nerve terminals from aberrant firings as suggested for the postsynaptic MNTB neurons (Brew and Forsythe, 1995). When nerve terminals are depolarized for a prolonged period, for example during anoxia, Kv1 channels will be activated and stabilize presynaptic terminals, thereby preventing generation of aberrant firings. It remains to be seen whether Kv1 channels shorten presynaptic action potentials in immature animals, which have broader presynaptic action potentials (Taschenberger and von Gersdorff, 2000).

\section{References}

Augustine GJ (1990) Regulation of transmitter release at the squid giant synapse by presynaptic delayed rectifier potassium current. J Physiol (Lond) 431:343-364.

Bielefeldt K, Rotter JL, Jackson MB (1992) Three potassium channels in rat posterior pituitary nerve terminals. J Physiol (Lond) 458:41-67.

Borst JGG, Helmchen F, Sakmann B (1995) Pre- and postsynaptic wholecell recordings in the medial nucleus of the trapezoid body of the rat. J Physiol (Lond) 489:825-840.

Brew HM, Forsythe ID (1995) Two voltage-dependent $\mathrm{K}^{+}$conductances with complementary functions in postsynaptic integration at a central auditory synapse. J Neurosci 15:8011-8022.

Campbell DL, Qu Y, Rasmusson RL, Strauss HC (1993) The calciumindependent transient outward potassium current in isolated ferret right ventricular myocytes. II. Closed state reverse use-dependent block by 4-aminopyridine. J Gen Physiol 101:603-626.

Coetzee WA, Amarillo Y, Chiu J, Chow A, Lau D, McCormack T, Moreno H, Nadal MS, Ozaita A, Pountney D, Saganich M, Vega-Saenz de Miera E, Rudy B (1999) Molecular diversity of $\mathrm{K}^{+}$channels. Ann NY Acad Sci 868:233-285.

Daniel H, Crepel F (2001) Control of $\mathrm{Ca}^{2+}$ influx by cannabinoid and metabotropic glutamate receptors in rat cerebellar cortex requires $\mathrm{K}^{+}$ channels. J Physiol (Lond) 537:793-800.

Dodson PD, Billups B, Rusznak Z, Szucs G, Barker MC, Forsythe ID (2003) Presynaptic Kv1.2 channels suppress synaptic terminal hyperexcitability following action potential invasion. J Physiol (Lond) 550:27-33.

Elezgarai I, Diez J, Puente N, Azkue JJ, Benitez R, Bilbao A, Knopfel T, Donate-Oliver F, Grandes P (2003) Subcellular localization of the voltage-dependent potassium channel Kv3.1b in postnatal and adult rat medial nucleus of the trapezoid body. Neuroscience 118:889-898.

Forsythe ID (1994) Direct patch recording from identified presynaptic terminals mediating glutamatergic EPSCs in the rat CNS, in vitro. J Physiol (Lond) 479:381-387.

Forsythe ID, Barnes-Davies M (1993) The binaural auditory pathway: excitatory amino acid receptors mediate dual time-course excitatory postsynaptic currents in the rat medial nucleus of the trapezoid body. Proc R Soc Lond B Biol Sci 251:151-157.

Garcia-Calvo M, Leonard RJ, Novick J, Stevens SP, Schmalhofer W, Kaczorowski GJ, Garcia ML (1993) Purification, characterization, and biosynthesis of margatoxin, a component of Centruroides margaritatus venom that selectively inhibits voltage-dependent potassium channels. J Biol Chem 268:18866-18874.

Geiger JPR, Jonas P (2000) Dynamic control of presynaptic $\mathrm{Ca}^{2+}$ inflow by fast-inactivating $\mathrm{K}^{+}$channels in hippocampal mossy fiber boutons. Neuron 28:927-939.

Harvey AL (1997) Recent studies on dendrotoxins and potassium ion channels. Gen Pharmacol 28:7-12.

Hopkins WF (1998) Toxin and subunit specificity of blocking affinity of three peptide toxins for heteromultimeric, voltage-gated potassium channels expressed in Xenopus oocytes. J Pharmacol Exp Ther 285:1051-1060.

Hu H, Shao L-R, Chavoshy S, Gu N, Trieb M, Behrens R, Laake P, Pongs O, Knaus HG, Ottersen OP, Storm JF (2001) Presynaptic $\mathrm{Ca}^{2+}$-activated $\mathrm{K}^{+}$channels in glutamatergic hippocampal terminals and their role in 
spike repolarization and regulation of transmitter release. J Neurosci 21:9585-9597.

Ishikawa T, Takahashi T (2001) Mechanisms underlying presynaptic facilitatory effect of cyclothiazide at the calyx of Held of juvenile rats. J Physiol (Lond) 533:423-431.

Jackson MB, Konnerth A, Augustine GJ (1991) Action potential broadening and frequency-dependent facilitation of calcium signals in pituitary nerve terminals. Proc Natl Acad Sci USA 88:380-384.

Kajikawa Y, Saitoh N, Takahashi T (2001) GTP-binding protein $\beta \gamma$ subunits mediate presynaptic calcium current inhibition by $\mathrm{GABA}_{\mathrm{B}}$ receptor. Proc Natl Acad Sci USA 98:8054-8058.

Katz B, Miledi R (1969) Tetrodotoxin-resistant electric activity in presynaptic terminals. J Physiol (Lond) 203:459-487.

Knaus H-G, Koch ROA, Eberhart A, Kaczrowski GJ, Garcia ML, Slaughter RS (1995) [ $\left.{ }^{125} \mathrm{I}\right]$ Margatoxin, an extraordinarily high affinity ligand for voltage-gated potassium channels in mammalian brain. Biochemistry 34:13627-13634.

Lambe EK, Aghajanian GK (2001) The role of Kv1.2-containing potassium channels in serotonin-induced glutamate release from thalamocortical terminals in rat frontal cortex. J Neurosci 21:9955-9963.

Lupardus PJ, Wilke RA, Aydar E, Palmer CP, Chen Y, Ruoho AE, Jackson MB (2000) Membrane-delimited coupling between sigma receptors and $\mathrm{K}^{+}$ channels in rat neurohypophysial terminals requires neither G-protein nor ATP. J Physiol (Lond) 526:527-539.

Magistretti J, Mantegazza M, Guatteo E, Wanke E (1996) Action potentials recorded with patch-clamp amplifiers: are they genuine? Trends Neurosci 19:530-534.

Mathie A, Wooltorton JRA, Watkins CS (1998) Voltage-activated potassium channels in mammalian neurons and their block by novel pharmacological agents. Gen Pharmacol 30:13-24.

Miura M, Belvisi MG, Stretton CD, Yacoub MH, Barnes PJ (1992) Role of $\mathrm{K}^{+}$channels in the modulation of cholinergic neural responses in guineapig and human airways. J Physiol (Lond) 455:1-15.

Robitaille R, Garcia ML, Kaczorowski GJ, Charlton MP (1993) Functional colocalization of calcium and calcium-gated potassium channels in control of transmitter release. Neuron 11:645-655.

Rudy B, Chow A, Lau D, Amarillo Y, Ozaita A, Saganich M, Moreno H, Nadal MS, Hernandez-Pineda R, Hernandez-Cruz A, Erisir A, Leonard C, Vega-
Saenz de Miera E (1999) Contributions of Kv3 channels to neuronal excitability. Ann NY Acad Sci 868:304-343.

Saitoh N, Hori T, Takahashi T (2001) Activation of the epsilon isoform of protein kinase $\mathrm{C}$ in the mammalian nerve terminal. Proc Natl Acad Sci USA 98:14017-14021.

Sakaba T, Ishikane H, Tachibana M (1997) $\mathrm{Ca}^{2+}$-activated $\mathrm{K}^{+}$current at presynaptic terminals of goldfish retinal bipolar cells. Neurosci Res 27:219-228.

Southan AP, Robertson B (2000) Electrophysiological characterization of voltage-gated $\mathrm{K}^{+}$currents in cerebellar basket and Purkinje cells: Kv1 and Kv3 channel subfamilies are present in basket cell nerve terminals. J Neurosci 20:114-122.

Sun X-P, Schlichter LC, Stanley EF (1999) Single-channel properties of BKtype calcium-activated potassium channels at a cholinergic presynaptic nerve terminal. J Physiol (Lond) 518:639-651.

Takahashi T, Forsythe ID, Tsujimoto T, Barnes-Davies M, Onodera K (1996) Presynaptic calcium current modulation by a metabotropic glutamate receptor. Science 274:594-597.

Tan YP, Llano I (1999) Modulation by $\mathrm{K}^{+}$channels of action potentialevoked intracellular $\mathrm{Ca}^{2+}$ concentration rises in rat cerebellar basket cell axons. J Physiol (Lond) 520:65-78.

Taschenberger H, von Gersdorff H (2000) Fine-tuning an auditory synapse for speed and fidelity: developmental changes in presynaptic waveform, EPSC kinetics, and synaptic plasticity. J Neurosci 20:9162-9173.

Wang L-Y, Kaczmarek LK (1998) High-frequency firing helps replenish the readily releasable pool of synaptic vesicles. Nature 394:384-388.

Weiger TM, Holmqvist MH, Levitan IB, Clark FT, Sprague S, Huang W-J, Ge P, Wang C, Lawson D, Jurman ME, Glucksmann MA, Silos-Santiago I, DiStefano PS, Curtis R (2000) A novel nervous system $\beta$ subunit that downregulates human large conductance calcium-dependent potassium channels. J Neurosci 20:3563-3570.

Weiser M, Vega-Saenz de Miera E, Kentros C, Moreno H, Franzen L, Hillman D, Baker H, Rudy B (1994) Differential expression of Shaw-related $\mathrm{K}^{+}$ channels in the rat central nervous system. J Neurosci 14:949-972.

Yazejian B, Sun X-P, Grinnell AD (2000) Tracking presynaptic $\mathrm{Ca}^{2+} \mathrm{dy}-$ namics during neurotransmitter release with $\mathrm{Ca}^{2+}$-activated $\mathrm{K}^{+}$channels. Nat Neurosci 3:566-571. 